\title{
Ouadi Sannur
}

Mines de silex pharaoniques

François Briois, Béatrix Midant-Reynes, Pierre-Antoine Beauvais et Mohamed Hamdan

\section{OpenEdition}

1 Journals

Édition électronique

URL : https://journals.openedition.org/baefe/2744

DOI : $10.4000 /$ baefe. 2744

ISSN : 2732-687X

Éditeur

ResEFE

\section{Référence électronique}

François Briois, Béatrix Midant-Reynes, Pierre-Antoine Beauvais et Mohamed Hamdan, « Ouadi Sannur » [notice archéologique], Bulletin archéologique des Écoles françaises à l'étranger [En ligne], Égypte, mis en ligne le 30 mai 2021, consulté le 01 juin 2021. URL : http://journals.openedition.org/ baefe/2744 ; DOI : https://doi.org/10.4000/baefe.2744

\section{Ce document a été généré automatiquement le 1 juin 2021.}

\section{cc) (i) (9)}

Le Bulletin archéologique des Écoles françaises à l'étranger est mise à disposition selon les termes de la Licence Creative Commons Attribution - Pas d'Utilisation Commerciale - Pas de Modification 4.0 International. 


\title{
Ouadi Sannur
}

\author{
Mines de silex pharaoniques \\ François Briois, Béatrix Midant-Reynes, Pierre-Antoine Beauvais et \\ Mohamed Hamdan
}

\section{NOTE DE L'AUTEUR}

Année de la campagne : 2020 (mission de terrain non réalisée)

Autorité nationale présente : Le ministère du Tourisme et des Antiquités (MoTA)

Numéro et intitulé de l'opération de terrain : 17133 - Ouadi Sannur

Composition de l'équipe scientifique : L'équipe était composée de François Briois (archéologue, EHESS, UMR 5608 TRACES), Béatrix Midant-Reynes (archéologue, CNRS, UMR 5608 TRACES), Pierre-Antoine Beauvais (doctorant, université Toulouse Jean Jaurès), Aurore Ciavatti (archéologue, Ifao), Mohamed Gaber (topographe, Ifao), Pr. Mohamed Hamdan (géologue, Faculté des sciences, Le Caire), Mohamed Hassan (restaurateur, Ifao), Valérie Le Provost (céramologue), Anita Quiles (laboratoire de datation, Ifao).

Partenariats institutionnels : Le projet bénéficie du soutien du fonds Khéops pour l'archéologie et du LabEx « Structurations des mondes sociaux » (SMS).

Données scientifiques produites :

https://www.archeonil.fr/index.php/les-chantiers/galala-nord

\section{Problématique et résultats des travaux antérieurs}

1 Les recherches entreprises au Ouadi Sannur depuis 2014 ont pour objectif l'étude d'un vaste complexe minier localisé sur la partie sud-ouest du massif du Galala nord. Les calcaires éocènes de ce plateau contiennent de riches ressources d'un silex gris calcédonieux de très belle qualité qui a fait l'objet d'une exploitation intensive depuis les premières dynasties jusqu'à l'Ancien Empire. Des carrières et des ateliers de 
production d'étendue variable ont été localisés à ce jour sur près de $1000 \mathrm{~km}^{2}$, dans une large zone entre Nil et mer Rouge, ainsi que des campements dont l'étude a été placée dans nos priorités.

Les mines constituent, en effet, des espaces d'interaction entre les populations du désert qui maîtrisent le milieu et les ressources et les groupes venus de la vallée (mineurs, tailleurs, administrateurs, soldats) pour en exploiter les richesses au profit d'une classe dominante, qui, de son côté, exerce sur cette exploitation une pression et une centralisation accrues. Comment les sites se positionnent-ils dans la géologie locale au sein d'un réseau de circulation et de communication qui joint la Vallée du Nil à la mer Rouge ? Quelles techniques de taille du silex ont été mises en œuvre pour produire à grande échelle des objets de qualité opérationnels, tant au plan économique (les lames) que symbolique (les couteaux)? Est-il possible de percevoir une dimension socio-économique au travers de l'étude des productions ? Existe-t-il des établissements relais ayant pu fonctionner comme des centres de redistribution? Ce sont ces questions qui constituent notre problématique et guident nos recherches sur le terrain.

En 2014, les travaux ont porté sur deux centres miniers importants (WS 100 et WS 118) et sur la fouille d'un ensemble de quatre bâtiments localisés près du ouadi Nikhaybar (WS 05), datés de l'Ancien empire. La campagne 2015 a été consacrée à la fouille et au relevé d'un nouveau campement localisé plus au sud (WS 010), sur lequel a pu être mis en évidence un atelier de production de lames et de couteaux bifaciaux. En 2016, nous avons repris et complété les observations dans le Ouadi Umm Nikhaybar et sondé les restes d'un nouveau campement identifié sur une terrasse du ouadi (WS 13). La mission a également été consacrée à des prospections sur de nouvelles mines repérées dans le Ouadi Abu Rimth et à la fouille d'un atelier de débitage de lames intact implanté à proximité immédiate d'une carrière d'extraction de silex (WS 328). Depuis 2017, les campagnes ont principalement été consacrées à la fouille du vaste campement WS 13 localisé au fond du Ouadi Umm Nikhaybar. Ce camp de mineurs, de $600 \mathrm{~m}^{2}$ de superficie, est composé de plusieurs groupes de bâtiments qui ont fonctionné en relation avec les exploitations de silex et, dans une moindre mesure, la collecte de jaspe rouge du ouadi Sannur. Ce site, daté entre la II ${ }^{\mathrm{e}}$ et la IV ${ }^{\mathrm{e}}$ dynastie est le complexe le plus important identifié à ce jour sur le plateau du Galala. Il est placé au cœur d'un vaste réseau de circulation entre la Vallée du Nil à la mer Rouge. La mission de terrain, prévue en octobre 2020, a dû être annulée en raison de la crise sanitaire. Les activités menées cette année se sont reportées sur des travaux d'étude et d'analyse sur la documentation de fouille.

\section{Traitement des données de l'atelier WS13-B-1381}

La fouille du campement WS 013, entre 2017 et 2019, a permis la découverte d'un très gros amas de taille du silex localisé dans la pièce $B$ du complexe architectural du ouadi Nikhaybar. La concentration de silex, couvrant la majeure partie de l'espace construit, correspond à une aire de production industrielle de lames qui a également été dédiée à la fabrication de couteaux bifaciaux et de manière plus discrète de disques à taille bifaciale correspondant à des préformes de bracelets. Cette concentration lithique, la seule d'importance découverte à ce jour à l'échelle du site, offre une occasion exceptionnelle de pouvoir étudier un atelier de taille intact daté de l'Ancien Empire. L'analyse technologique de tous les déchets de taille récoltés au sein de cette aire de 
production spécialisée permet de dégager une vision générale de l'ensemble lithique par l'examen des chaînes opératoires, de formuler des hypothèses sur les techniques utilisées et d'aborder des questions d'ordre socio-économiques. Ces observations, jusqu'à présent inédites en Égypte, renseignent sur les modes de production et d'organisation des équipes d'artisans en lien avec la fabrication des outillages de silex. L'année 2020 a été consacrée à la mise au net de toute la documentation photographique et des relevés de terrain, au traitement et à l'interprétation des données en vue de la rédaction d'un article de synthèse.

\section{Datations 14C}

Plusieurs séries d'échantillons de charbons de bois provenant des foyers du site WS 13 ont été exportées au Caire pour être traités au laboratoire de datation 14C de l'Ifao. Les résultats obtenus jusqu'à présent indiquent que le site était occupé entre 2900 et $2450 \mathrm{av}$. J.-C., ce qui correspond très probablement à la période d'exploitation des mines du ouadi Sannour. Il est difficile de déterminer si l'occupation a été continue jusqu'au début de l'Ancien Empire où la production a atteint un sommet. Le fait est que les traits forts les plus apparents, tant au plan de la production lithique que des céramiques, concordent avec ceux de la IV ${ }^{\mathrm{e}}$ dynastie.

Une nouvelle série d'échantillons prélevés en 2019 et toujours en cours de traitement devrait offrir de nouveaux résultats pour consolider le modèle chronologique.

\section{Les formations de silex du Galala nord}

7 La description des formations géologiques en relation avec les contextes miniers, de la partie sud-occidentale du plateau du Galala nord, a été amorcée en 2018 par le professeur Mohamed Hamdan, qui a produit un rapport scientifique. La réalisation de lames minces et l'analyse d'échantillons géologiques ont été effectuées au laboratoire de géologie de la faculté des sciences du Caire. Les résultats constituent un point de départ pour la caractérisation des différentes variétés de silex en présence. Ce référentiel permettra de reconstruire les routes d'échange du silex par comparaison formelle des matériaux géologiques avec les très riches collections provenant des habitats et des nécropoles où se trouvent les objets les plus prestigieux.

\section{Communications scientifiques}

- François Briois, Béatrix Midant-Reynes, «Récentes découvertes en Égypte: les mines et exploitations de silex du Galala nord (désert oriental) », conférence Archéonil à l'École du Louvre le 5 février 2020.

- François Briois, Béatrix Midant-Reynes, « Les mines du ouadi Sannour (désert oriental). Des ateliers de production d'outils silex de la fin du Prédynastique à l'Ancien Empire", conférence au cercle lyonnais d'égyptologie Victor Loret, le 11 mars 2020. 


\section{Publications}

- François Briois, Béatrix Midant-Reynes, Frédéric Guyot, «The Flint Mines of North Galala (Eastern Desert)», in E.Christinia Köhler, Nora Kuch, Friederike Junge, Ann-Kathrin Jeske (éd.), Egypt at its Origins 6: Proceedings of the Sixth International Conference on Predynastic and Early Dynastic Egypt, Vienna, 10th-15th September 2017, OLA, Louvain, Paris, Bristol, 2020, p. 65-82.

- François Briois, Béatrix Midant-Reynes, «Ouadi Sannur ", Bulletin archéologique des Écoles françaises à l'étranger, 2020, article en ligne sur OpenEdition Journals, https:// journals.openedition.org/baefe/955, consulté le 16 mai 2021.

INDEX

Thèmes : IFAO

Année de l'opération : 2020

\section{AUTEURS}

\section{FRANÇOIS BRIOIS}

Archéologue, EHESS, UMR 5608 TRACES

\section{BÉATRIX MIDANT-REYNES}

Archéologue, CNRS, UMR 5608 TRACES

\section{PIERRE-ANTOINE BEAUVAIS}

Doctorant, université Toulouse Jean Jaurès

\section{MOHAMED HAMDAN}

Géologue, Faculté des sciences, Le Caire 